\title{
MicroRNA 144 inhibits cell migration and invasion and regulates inflammatory cytokine secretion through targeting toll like receptor 2 in non-small cell lung cancer
}

Rong $\mathrm{Pu}^{1}$, Meicen $\mathrm{Pu}^{2}$, Haohai Huang ${ }^{3}$, Yejia Cui ${ }^{1}$

\begin{abstract}
1Department of Laboratory, The Third People's Hospital of Dongguan, Dongguan, Guangdong, China

${ }^{2}$ Department of Clinical Medicine, Fujian Medical University, Fuzhou, Fujian, China ${ }^{3}$ Department of Education and Science, The Third People's Hospital of Dongguan, Dongguan, Guangdong, China
\end{abstract}

Submitted: 24 February 2018; Accepted: 14 June 2018

Online publication: 10 March 2020

Arch Med Sci 2021; 17 (4): 1028-1037

DOI: https://doi.org/10.5114/aoms.2020.93084

Copyright (c) 2020 Termedia \& Banach

\section{Abstract}

Introduction: MicroRNAs (miRNAs) are endogenous small noncoding RNA molecules involved in modulation of cancer progression. Here, we investigated the possible role of miR-144 in non-small cell lung cancer (NSCLC) development.

Material and methods: The expression of miR-144 and TLR2 in NSCLC tissue and cell lines was determined by quantitative real-time PCR (qPCR). The TargetScan database was used to predict potential target genes of miR-144. Luciferase assay was used to verify the interaction between TLR2 and miR-144. TLR2 protein expression was measured by western blot. The secretion of interleukin (IL)-1 $\beta$, IL- 6 and IL-8 in A549 cells was detected by an ELISA kit. Cell migration and invasion were evaluated by wound healing assay and transwell assay, respectively.

Results: Our results showed that miR-144 was downregulated in NSCLC tissue and cell lines when compared with the normal tissues and cell line $(p<0.05)$. The protein level of TLR2 in NSCLC tissue and cell lines was significantly higher than that in normal lung tissues. Dual luciferase reporter gene assay showed that miR-144 could bind to the 3'UTR of TLR2 specifically. Up-regulation of miR-144 significantly decreased the expression of TLR2. Up-regulation of miR-144 or down-regulation of TLR2 could decrease cell migration, invasion and secretion of IL-1 $\beta$, IL- 6 and IL-8 in A549 cells. Moreover, overexpression of TLR2 rescued the inhibitory effects of miR-144 on migration, invasion and inflammatory factor secretion of A549 cells.

Conclusions: miR-144 could inhibit the migration, invasion and secretion of IL-1 $\beta$, IL- 6 and IL- 8 through downregulation of TLR2 expression in A549 cells.

Key words: non-small cell lung cancer, MiR-144, TLR2, inflammation.

\section{Introduction}

Lung cancer is one of the leading causes of cancer-related death worldwide [1]. The two main types of lung cancer are small cell lung cancer (SCLC) and non-small cell lung cancer (NSCLC). Non-small cell lung

\author{
Corresponding authors: \\ Rong Pu \\ Yejia Cui \\ Department of Laboratory \\ The Third People's Hospital \\ of Dongguan \\ No. 1, Xianglong Road \\ Shilong town \\ Dongguan 523326 \\ Guangdong, China \\ Phone: +86-0769-81368090 \\ E-mails: purong2017@126.com \\ (Rong Pu); cuiyejia1@126.com \\ (Yejia Cui)
}


cancer represents approximately $85 \%$ of lung cancer cases [2]. Despite advances in diagnosis and treatment of lung cancer, NSCLC still has a high mortality rate globally. The 5 -year overall survival rate of NSCLC is lower than 15\% [3]. Thus, there is a great need to elucidate the molecular mechanisms underlying development and progression of NSCLCS.

Tumor-related inflammation is a hallmark of malignant cancer, which modulates tumor initiation and development [4]. Cancer can induce an inflammatory response [5]. Chronic inflammation promotes tumor progression by stimulating angiogenesis, epithelial-to-mesenchymal transition (EMT) and evasion from apoptosis [6]. Lung is a specific organ which is prone to form an inflammatory microenvironment [7]. As a kind of pattern recognition receptors, Toll-like receptors (TLRs) not only regulate innate and adaptive immunity, but also modulate production of inflammatory cytokines [8]. Activation of TLRs can lead to the secretion of pro-inflammatory cytokines, such as interleukin (IL)-1 $\beta$, IL- 6 and IL-8 [9]. Furthermore, recent studies have reported that NSCLC is associated with inflammatory cytokines that affect cell migration, invasion and tumor chemoresistance [7, 9]. For instance, IL-17 promoted metastasis of non-small-cell lung cancer cells [10]. IL-6-mediated inflammation may contribute to NSCLC-related morbidity and mortality [11], which make it a therapeutic target of NSCLC. These observations prompted us to study whether TLRs are potential targets of NSCLC therapy.

MicroRNAs (miRNAs) are a class of $\sim 22$ nucleotide (nt) small noncoding RNAs that negatively regulate gene expression via either mRNA degradation or interfering mRNA translation [12]. miRNAs play a regulatory role in many biological processes including cell apoptosis, differentiation and migration [13]. Recent studies have indicated that miRNAs can be considered as oncogenes and tumor suppressors [14].

MicroRNA 144 (miR-144) is a tumor suppressor in various types of cancers, including gastric cancer, bladder cancer and colorectal carcinoma [1518]. However, its roles in TLR-mediated NSCLCS remain unclear. In this study, we found that miR-144 was down-regulated and the levels of TLR2 were significantly increased in NSCLC patients, compared to the paired non-tumor lung tissue. Bioinformatics analyses and the luciferase reporter assay revealed that miR-144 could directly bind to the 3'UTR of TLR2. miR-144 inhibited NSCLC cell migration, invasion and inflammatory cytokine secretion through targeting TLR2, which suggests that reinstating miR-144 expression might be usefully therapeutic treatment for NSCLCS.

\section{Material and methods}

\section{Patient samples and cell lines}

Twenty-eight human NSCLCs and corresponding normal tissues $(6 \mathrm{~cm}$ away from the primary tumor) were collected during tumor surgery at The Third People's Hospital of Dongguan. The tissues were frozen and stored in liquid nitrogen until RNA extraction.

This study was approved by the Medical Ethics and Human Clinical Trial Committee at The Third People's Hospital of Dongguan. Written informed consent was signed by each patient.

Normal lung bronchus epithelial cell line BEAS-2B and NSCLC cell lines (H460, H520, SK-MES-1, H157 and A549) were obtained from American Type Culture Collection (ATCC, USA). These above cell lines were maintained in Dulbecco's modified Eagle's medium (DMEM) with 10\% FBS, $100 \mathrm{U} / \mathrm{ml}$ penicillin, and $100 \mathrm{mg} / \mathrm{ml}$ streptomycin (Invitrogen, Carlsbad, CA, USA) at $37^{\circ} \mathrm{C}$ within $5 \% \mathrm{CO}_{2}$.

\section{Plasmid constructions and transfection}

To overexpress miR-144, the cells were transfected with $0.05 \mu \mathrm{M}$ miR-144 mimics or miR-NC control (RiboBio, Guangzhou, People's Republic of China) using Lipofectamine 3000 (Thermo Fisher Scientific) according to the manufacturer's instructions. To knock down TLR2, the lentivirus containing the shRNAs of TLR2 and the scramble control were purchased from Santa Cruz. To construct a TLR2-expressing plasmid, the fulllength human TLR2 coding region lacking the entire 3'-UTR was amplified by PCR and cloned into the pcDNA3.1(+) vector (Invitrogen). Cells were co-transfected with miR-144 mimic $(0.05 \mu \mathrm{M})$ and pcDNA3.1-TLR2 plasmid or pcDNA3.1(+) vector $(1 \mu \mathrm{g})$ using Lipofectamine 3000 (Thermo Fisher Scientific) according to the manufacturer's instructions.

\section{Dual luciferase assays}

A wild-type 3'-UTR fragment of TLR2 CDNA (WT) was amplified using PCR and cloned into the pmirGLO dual-luciferase miRNA target expression vector (Promega, Madison, WI, USA). The mutant variant of TLR2 3'-UTR (MUT) was generated based on WT by mutating eight nucleotides that potentially bind to miR-144. A549 cells were cotransfected with miR-144 mimics or miR-NC and WT or Mut vectors. After transfection for $48 \mathrm{~h}, 2 \times 10^{4}$ cells per well were seeded in a 24-well plate. At $80-90 \%$ confluence, luciferase activity was assayed using the Dual-Luciferase Reporter 1000 Assay System (Promega, Madison, USA). 


\section{RNA isolation and quantitative real-time} polymerase chain reaction

Total RNA was extracted from lung tumor tissues and cell lines using TRIzol reagent (Invitrogen, Carlsbad, CA, USA) according to the manufacturer's instructions. MiRNA cDNA was generated using the PrimeScript miRNA cDNA Synthesis kit (Takara, Dalian, China). The expression of mature miR-144 was assayed using TaqMan MicroRNA Assays (Applied Biosystems, Foster City, CA, USA). U6 snRNA was amplified as an internal control. To detect the mRNA level of TLR2, CDNA was reverse transcribed with the RevertAid First Strand cDNA Synthesis Kit (Thermo Scientific, USA). Real-time quantitative polymerase chain reaction ( $q P C R)$ was performed using SYBR Premix Ex Taq (Takara Bio, Dalian, China). mRNA levels were normalized to the level of $\beta$-actin. The primers used were as follows as described in a previous study [19]: TLR2 forward primer, 5'-GCC AAA GCT TTG ATT GAT TGG-3'; TLR2 reverse primer, 5'-TTG AAG TTC TCC AGC TCC TG-3'; $\beta$-actin forward primer, 5'-CCC CAG GCA CCA GGG CGT GAT-3'; and $\beta$-actin reverse primer, 5'-GGT CAT CTT CTC GCG GTT GGC CTT GGG GTT-3'. The relative expression of each gene was calculated using the $2^{-\Delta \Delta c t}$ method.

\section{Protein extraction and western blot}

Total proteins were extracted using a RIPA lysis buffer (Beyotime Biotech Inc., China) according to the manufacturer's instructions. Protein concentrations were quantified using the BCA method (Beyotime, Jiangsu, China). Proteins $(40 \mu \mathrm{g})$ were fractionated by $10 \%$ SDS-polyacrylamide gel (SDS-PAGE), and then electrotransferred to nitrocellulose membranes (Whatman, Maidstone, UK). The membrane was blocked with $5 \%$ nonfat milk for $10 \mathrm{~min}$ and then incubated overnight at $4^{\circ} \mathrm{C}$ with primary antibodies against TLR2 (Santa Cruz, USA) and $\beta$-actin (Santa Cruz), respectively. The membranes were washed three times and then incubated with horseradish peroxidase-conjugated secondary antibodies for $1 \mathrm{~h}$. Protein bands were examined by visualization with the Enhanced Chemiluminescence Plus Kit (GE Healthcare). The relative protein expression was normalized to $\beta$-actin.

\section{Wound healing assay}

Cell migration was examined by scratch wound healing assay. After transfection for $48 \mathrm{~h}$, cells were seeded in six-well plates at a density of $1 \times 10^{6}$ cells/well. To generate scratch wounds, confluent cell monolayers were then scraped with a $10 \mu \mathrm{l}$ pipette tip and washed twice with PBS buffer. Cells were incubated at $37^{\circ} \mathrm{C}$ for $48 \mathrm{~h}$. The spread of wound closure was observed and photographed under a microscope. The wound areas were calculated by NIH ImageJ software (Bethesda, MA, USA).

\section{Transwell invasion assays}

Cell invasion was detected by transwell invasion assays. $5 \times 10^{4}$ cells in serum-free media were added into the upper chamber of the BioCoat Invasion Chambers (Becton-Dickinson Biosciences, Bedford, MA, USA). The lower chamber was filled with $1 \mathrm{ml}$ of DMEM with $1 \%$ FBS. After $24 \mathrm{~h}$, the cells inside the upper chamber were removed. The invaded and migrated cells on the lower membrane were fixed and stained with $4 \%$ polyformaldehyde (PFA) and $0.2 \%$ crystal violet, imaged, and counted for 10 random $\times 100$ fields per well.

\section{Detection of inflammatory factors}

After transfection for $48 \mathrm{~h}$, cells were starved in serum-free medium for $24 \mathrm{~h}$. The cell-cultured conditioned media were collected, and were centrifuged at $3000 \mathrm{rpm}$ for $10 \mathrm{~min}$. The supernatant media were transferred to a new tube. The levels of IL-1 $\beta$, IL- 6 and IL- 8 in above supernatants were detected using a Human Quantikine ELISA kit (R\&D Systems, Minneapolis, MN) according to the manufacturer's instructions.

\section{Statistical analyses}

Quantitative data were expressed as mean \pm standard deviation (SD). The statistical analysis was carried out by ANOVA post hoc tests (Dunnett) using SPSS 19.0 Software. A $p$-value less than 0.05 was considered significant.

\section{Results}

MicroRNA 144 is down-regulated in nonsmall cell lung cancer tissues and cell lines

To evaluate the expression and role of miR144 and TLR2 in NSCLC development, we detected the expression of miR-144 and TLR2 in 28 pairs of NSCLC tissues and the corresponding normal lung tissues using qPCR. The results showed that miR-144 was significantly down-regulated in NSCLC tissues, compared with that in the corresponding normal lung tissues (Figure 1 A). Furthermore, the expression level of miR-144 was tested in five NSCLC cell lines. As shown in Figure $1 \mathrm{C}$, the expression levels of miR-144 were markedly decreased in these NSCLC cells, compared with the level in the normal cell line BEAS-2B. In addition, we observed that TLR2 was significantly up-regulated in NSCLC tissues and cell lines compared with the corresponding normal lung tissues and normal cell line BEAS-2B, respectively (Figures $1 \mathrm{~B}, \mathrm{D}$ ). Thus, these observations suggested that miR-144 might play negative roles in 

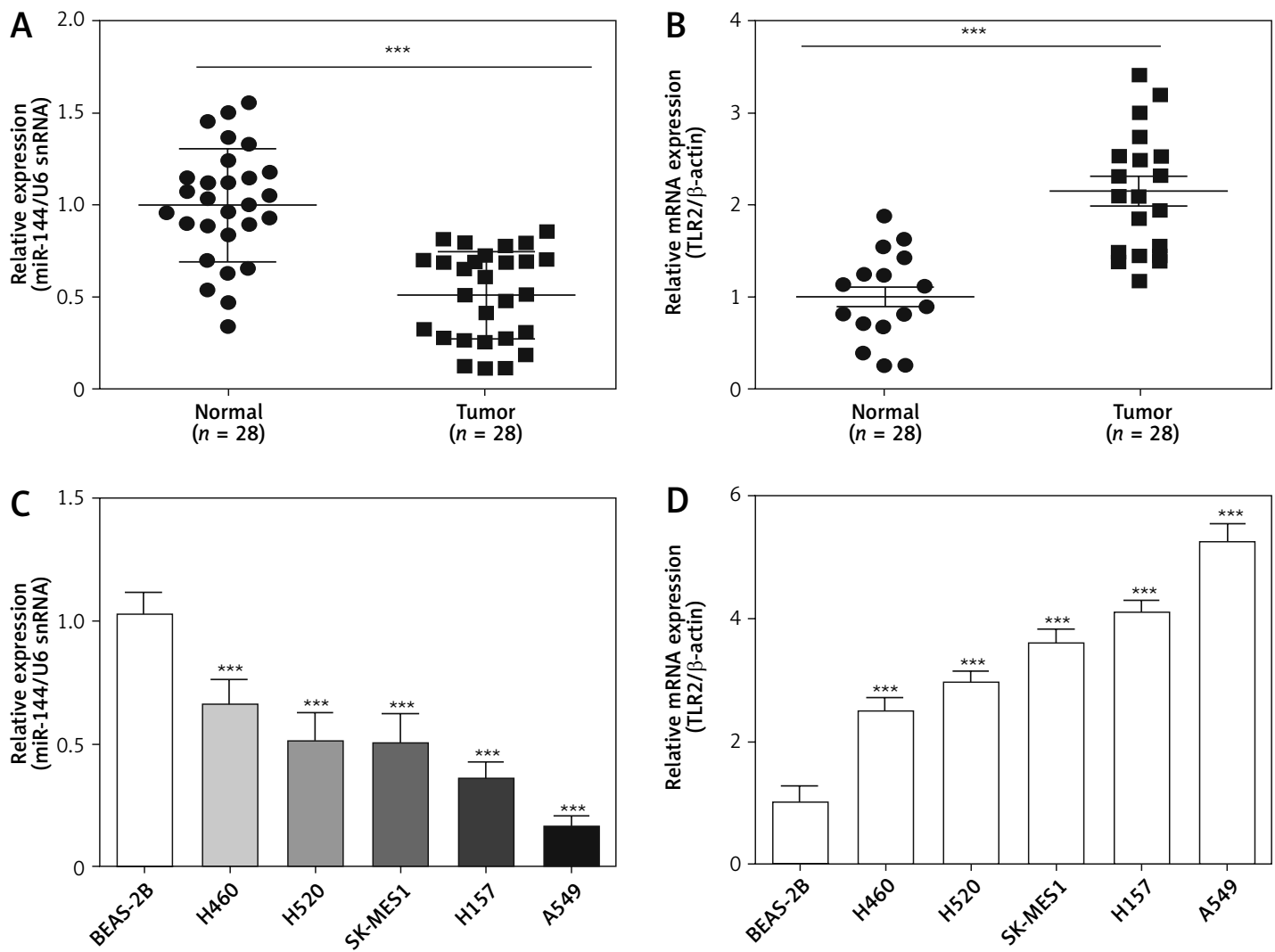

Figure 1. Expression of miR-144 and TLR2 in non-small cell lung cancer (NSCLC) tissues and cell lines. A, B - Expression levels of miR-144 and TLR2 in 28 pairs of NSCLC tissues and their corresponding normal lung tissues were measured by qPCR. U6 snRNA and $\beta$-actin were used as internal controls, respectively. C, D - Expression levels of miR-144 and TLR2 in normal lung epithelial cell line (BEAS-2B) and 5 NSCLC cell lines (H460: large cell cancer cell line, H520: squamous cancer cell lines; A549: adenocarcinoma cell line; H157, SK-MES1) were measured by qPCR. ${ }^{* * *} P<0.001$. (vs. normal or BEAS-2B groups)

NSCLC development. The opposite expression trend between miR-144 and TLR2 suggested their opposite role in NSCLC progression.

miR-144 inhibits non-small cell lung cancer cell migration and invasion in vitro

To further study the role of miR-144 in the progression of NSCLC, we transfected A549 cells with miR-144 mimic or miR-Control. As shown in Figure 2 A, miR-144 was successfully overexpressed in A549 cells. We examined whether miR-144 could also inhibit cell migration and invasion in NSCLC using wound healing assay and transwell migration assay, respectively. The results showed that overexpression of miR-144 dramatically suppressed the migratory and invasive capacity of A549 cells (Figures 2 B, C). Therefore, inhibitory effects on NSCLC cell migration and invasion in vitro suggested that miR-144 might suppress NSCLC growth and metastasis.

\section{TLR2 is a target of miR-144}

To investigate the mechanisms by which miR144 performs its function, we found that TLR2 is a potential candidate of miR-144 as predicted by TargetScan. The complementary sequence of miR-144 was identified in 3'UTR of TLR2 (Figure $3 \mathrm{~A}$ ). To confirm whether TLR2 is the direct downstream target of miR-144, luciferase reporter assay was performed to detect the interaction between miR-144 and 3'UTR of TLR2. The sequences of wild type (WT) and mutant (MUT) TLR2 3'UTR are shown in Figure 3 A. Furthermore, we observed that up-regulation of miR-144 significantly decreased the relative luciferase activity of WT TLR2 3'UTR in A549 cells (Figure 3 B). However, luciferase activity was not affected by transfection of MUT in miR-144 overexpressed A549 cells (Figure 3 B). Taken together, these results suggest that miR-144 directly targets 3'UTR of TLR2.

\section{miR-144 inhibits TLR2 expression and inflammatory cytokine secretion of non-small cell lung cancer cells}

To further investigate whether miR-144 targets TLR2 and decreases its expression, we detected the expression of TLR2 in miR-144 over- 


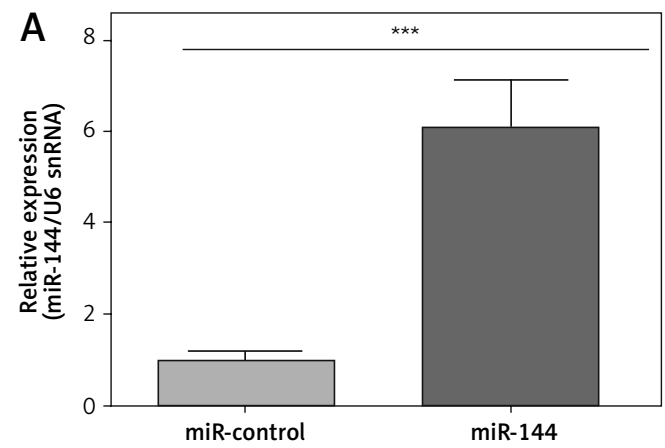

B
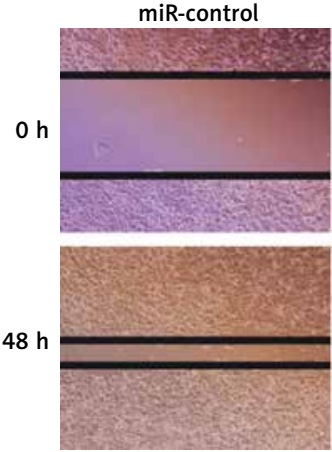

C
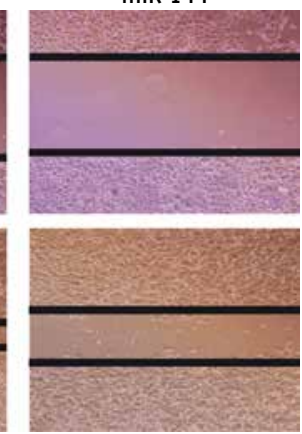

miR-control

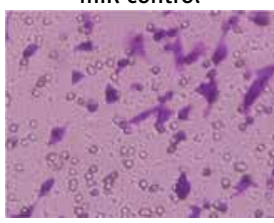

miR-144

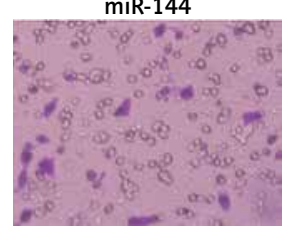

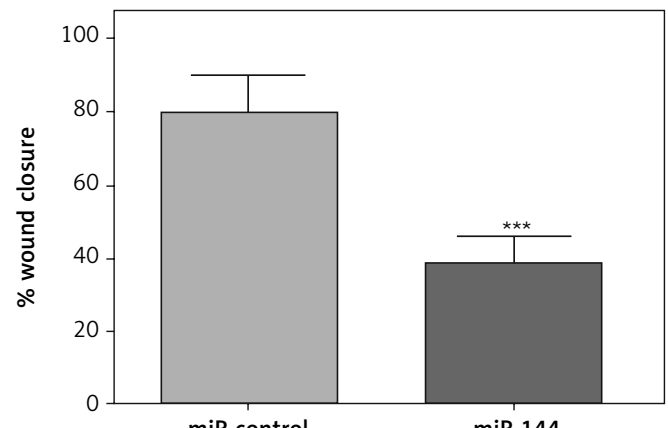

miR-control

miR-144

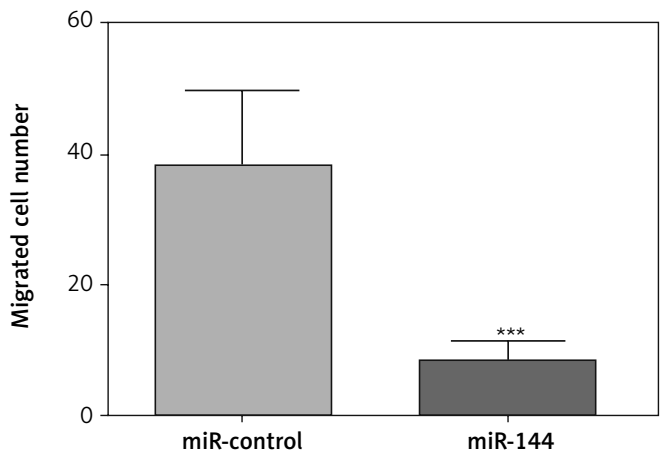

Figure 2. Overexpression of miR-144 inhibits cell proliferation, migration and invasion of A549 cells. A - A549 cells were infected with miR-144 mimic or miR-control, and the expression of miR-144 was detected by qPCR. B - Cell migration was detected by wound healing assay. C - Cell invasion was detected by transwell invasion assay. ${ }^{* *} P<0.001$ (vs. miR-control group)

A

Position 246-252 of TLR2 3' UTR (WT) 5'...CAUAAGUCUAUUACUGAUAUCU...3'

miR-144

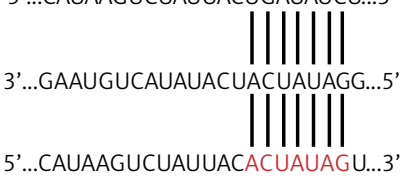

TLR2 3' UTR mutation (MUT) 5'...CAUAAGUCUAUUACACUAUAGU....3'

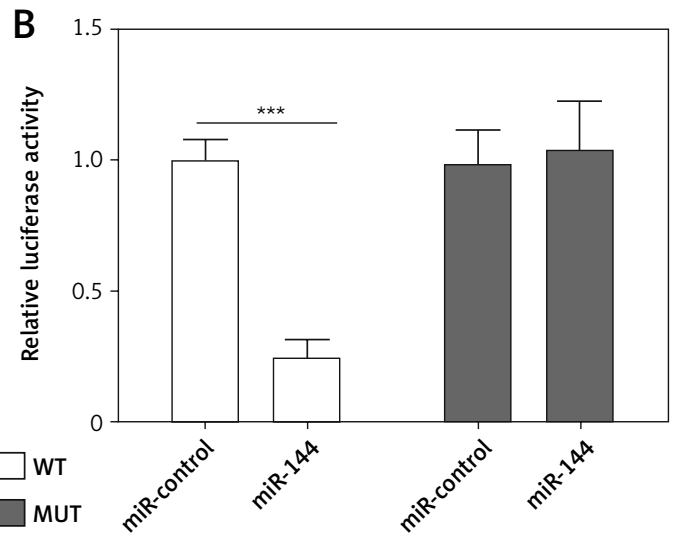

Figure 3. TLR2 is a downstream target of miR-144. A - Putative binding sequences of miR-144 in the TLR2 3'UTR. Mutation was generated in the TLR2 3'UTR by mutating 7 nt that is recognized by miR-144. Either wild-type (WT) or mutant (Mut) TLR2 3'UTR was subcloned into the dual-luciferase reporter vector. B - A549 cells were cotransfected with miR-144 mimic and luciferase reporter containing the TLR2 3'-UTR (WT) or a mutant (Mut). Luciferase activities were measured $48 \mathrm{~h}$ after transfection. ${ }^{* *} P<0.001$ 


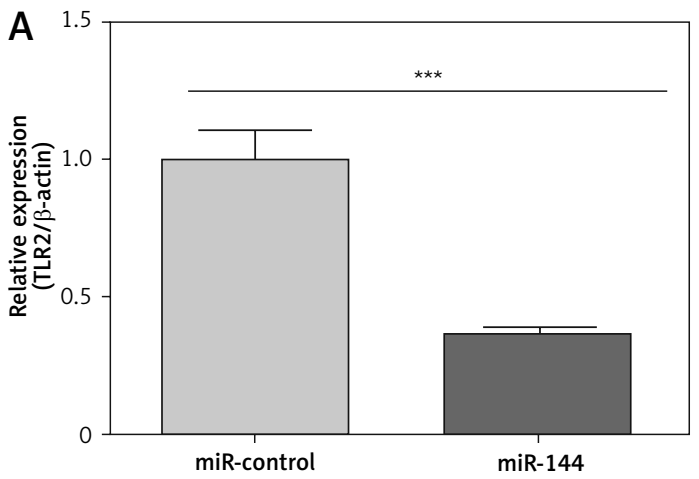

B
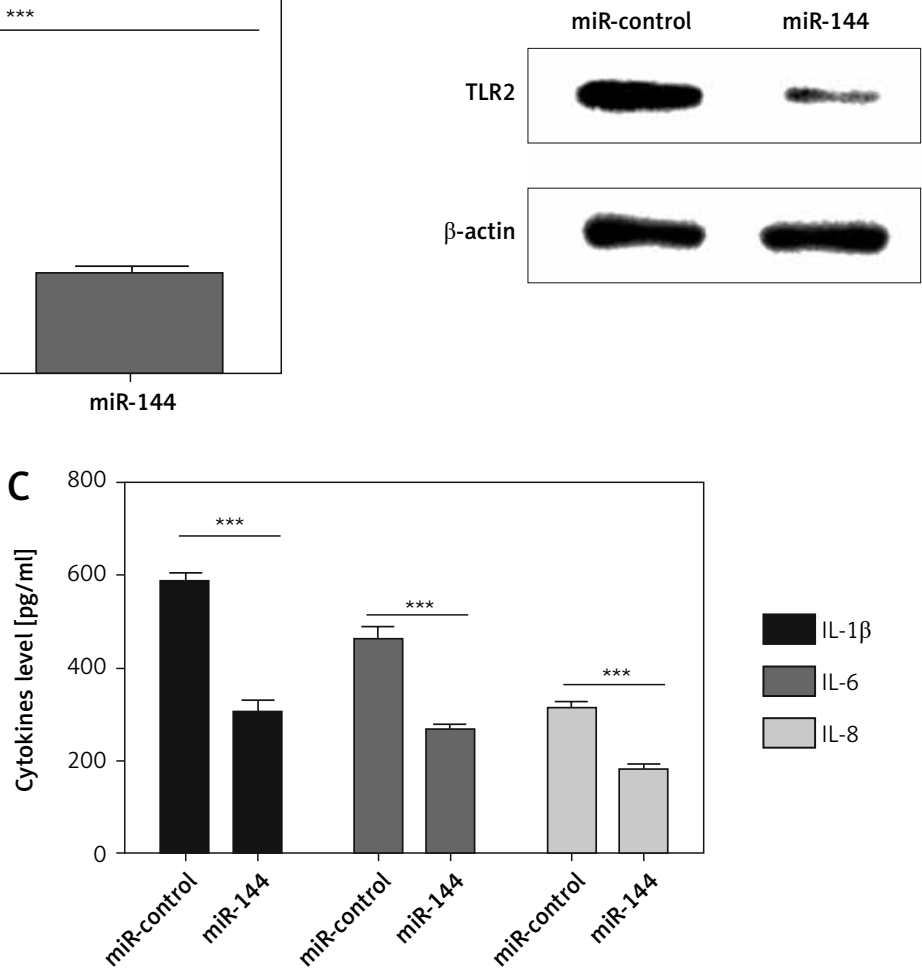

Figure 4. miR-144 regulates expression of TLR2 and inflammatory cytokine secretion of A549 cells. A549 cells were infected with miR-144 mimic or miR-control. A, B - mRNA level and protein level of TLR2 were detected by qPCR (A) and western blotting (B), respectively. C - Inflammatory cytokine secretion was detected by ELISA after transfection with miR-144 mimic for $48 \mathrm{~h}$. ${ }^{* \star *} P<0.001$

expressed A549 cells. Our results showed that the mRNA and protein levels of TLR2 were significantly down-regulated by miR-144 overexpression (Figures $4 \mathrm{~A}, \mathrm{~B}$ ). As is well known, TLR2 is an important regulator of inflammatory cytokine secretion [20]. Therefore, we further explored whether miR-144 could also inhibit inflammatory cytokine secretion of NSCLC cells. The secretion of IL-1 $\beta$, IL- 6 and IL- 8 was detected in miR-144 overexpressed $A 549$ cells. We observed that overexpression of miR-144 dramatically decreased the secretion of IL-1 $\beta$, IL- 6 and IL- 8 in A549 cells compared with their corresponding controls (Figure $4 \mathrm{C}$ ). Taken together, these results suggest that miR-144 can suppress inflammatory cytokine production of NSCLC cells in vitro.

\section{Knocking down TLR2 prevents migration} and invasion and inflammatory cytokine secretion of non-small cell lung cancer cells

To further investigate the role of TLR2 in NSCLC development, we knocked down the expression of TLR2 by its shRNAs. As shown in Figure $5 \mathrm{~A}$, the protein level of TLR2 was successfully down-regulated in A549 cells. We further performed wound healing assay and transwell invasion assay to ex- amine the effects of TLR2 on NSCLC cell migration and invasion, respectively. The results showed that down-regulation of TLR2 dramatically prevented migration and invasion of A549 cells compared with their corresponding controls (Figures $5 \mathrm{~B}, \mathrm{C}$ ). In addition, we found that down-regulation of TLR2 also decreased the secretion of IL-1 $\beta$, IL- 6 and IL- 8 in A549 cells compared with their corresponding controls (Figure $5 \mathrm{D}$ ). Therefore, TLR2 promoted NSCLC cell migration and invasion in vitro, which confirmed that miR-144 and TLR2 play opposite roles in NSCLC development in vitro.

\section{TLR2 is involved in miR-144-induced inhibitory effects on migration, invasion and inflammatory cytokine secretion of non-small cell lung cancer cells}

To further indicate whether miR-144 exerts its tumor inhibitory effects through targeting TLR2, we overexpressed TLR2 in miR-144 overexpressed A549 cells and detected the abilities of cell migration, invasion and inflammatory cytokine secretion. Firstly, TLR2 was overexpressed in A549 cells by cotransfection with miR-144 mimic and TLR2 lacking 3'UTR vector (TLR2) or control vector (Vector). The results showed that TLR2 overexpression did not affect 
A

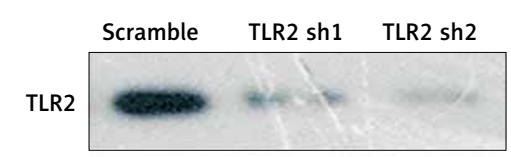

$\beta$-actin

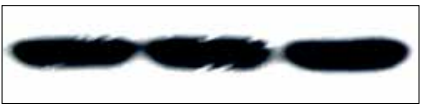

C
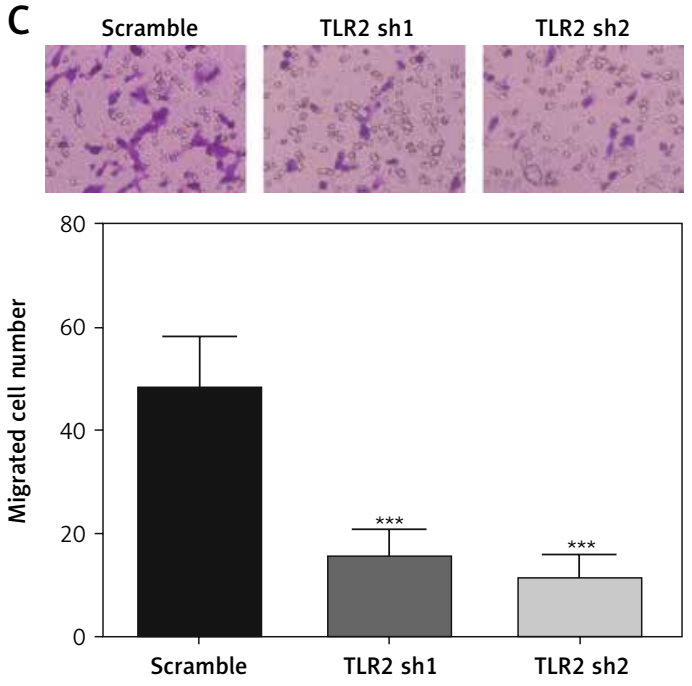

D

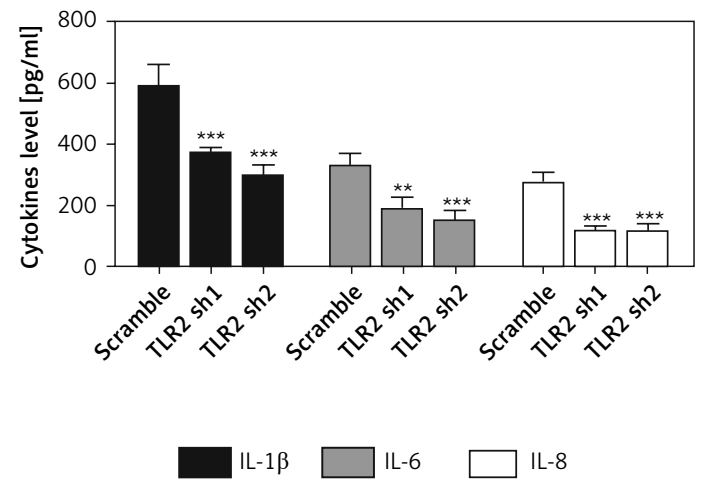

the expression of miR-144 (Figure 6 A). Western blotting analysis confirmed that TLR2 was overexpressed in A549 cells that overexpressed miR-144 (Figure 6 B). As expected, we found that the inhibitory effects of miR-144 on cell migration, invasion and inflammatory cytokine secretion were antagonized by TLR2 overexpression (Figures 6 C-E). Taken together, these data indicated that miR-144 played an inhibitory role in NSCLC development by targeting TLR2.

\section{Discussion}

Variations of miRNAs' expression are involved in almost all cancer biological functions, such as cell migration, invasion and apoptosis, which suggests that they can regulate tumor behavior as either tumor suppressors or oncogenes [14].
B
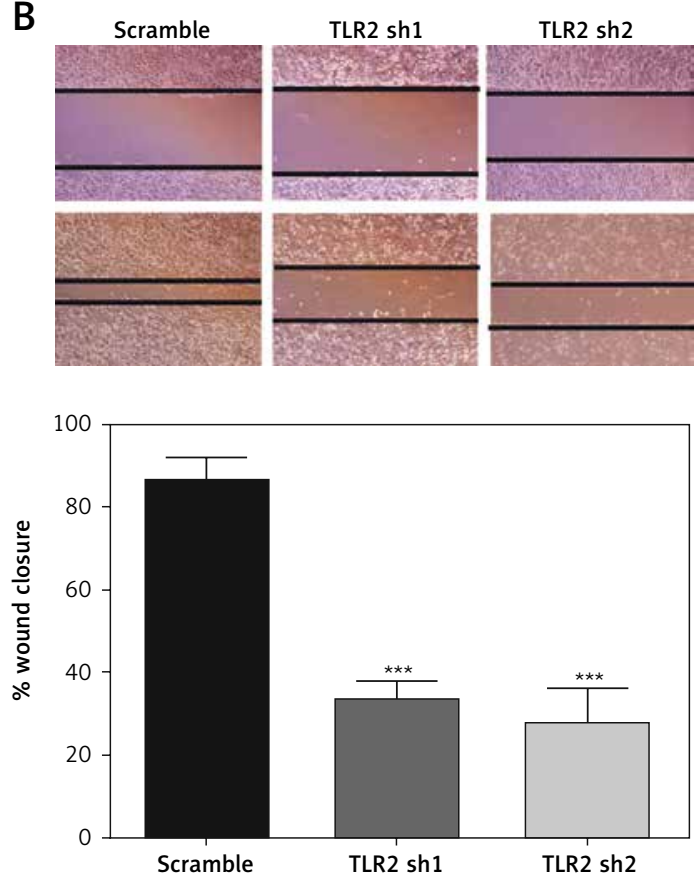

Figure 5. Down-regulation of TLR2 inhibits cell proliferation, migration, invasion and inflammatory cytokine release of A549 cells. A549 cells were transfected with TLR2 shRNAs or scramble control vector. After transfection for $48 \mathrm{~h}$. A - Expression of TLR2 was detected by western blot. B - Cell migration was detected by wound healing assay. C - Cell invasion was detected by transwell invasion assay. D - Inflammatory cytokine secretion was detected by ELISA. ${ }^{* \star} P<0.01,{ }^{* * *} p<0.001$, vs. scramble group

The alterations of microRNA expression in lung cancer have been identified [21]. Compared with the normal tissue counterparts, the expression of miR-21, miR-210 miR-191, miR-155, miR-146, miR-203, miR-17-3p and miR-205 was upregulated in lung cancer, whereas the levels of miR-143, miR125a, miR-33, miR-224, miR-126, miR-107 and miR185 were decreased [22]. In the present study, we found that miR-144 was down-regulated in NSCLC tissues and cell lines, which was consistent with previous results [21]. Overexpression of miR-144 could effectively prevent NSCLC cell motility, invasion and proinflammatory cytokine release in vitro. These results suggest that miR-144 might be a novel tumor suppressor.

miRNAs regulate biological functions by inhibiting downstream gene expression [23]. It has 
A

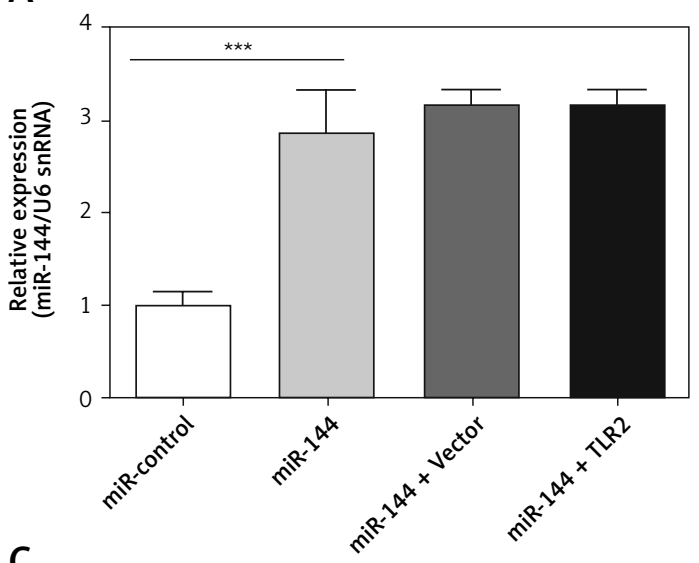

C

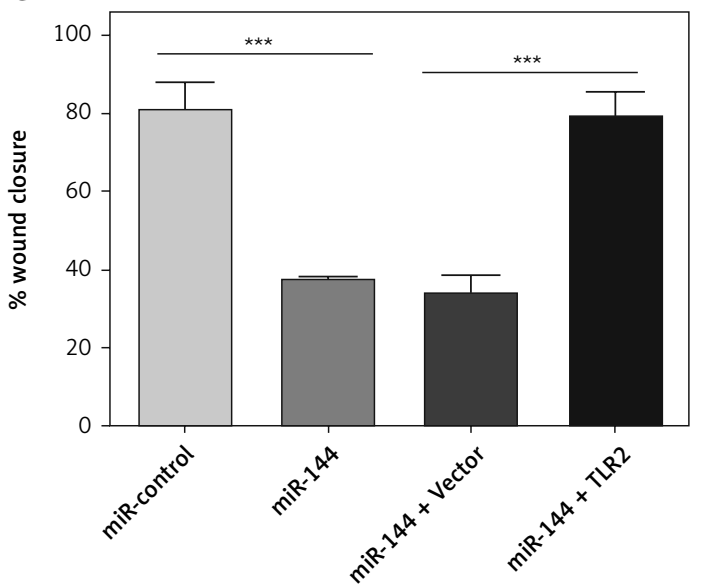

B

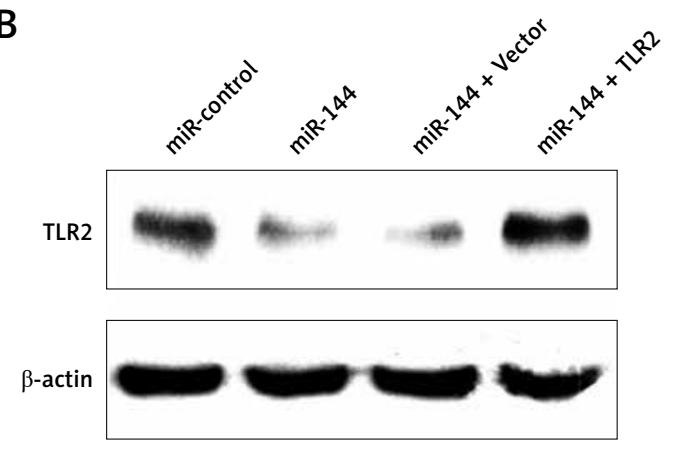

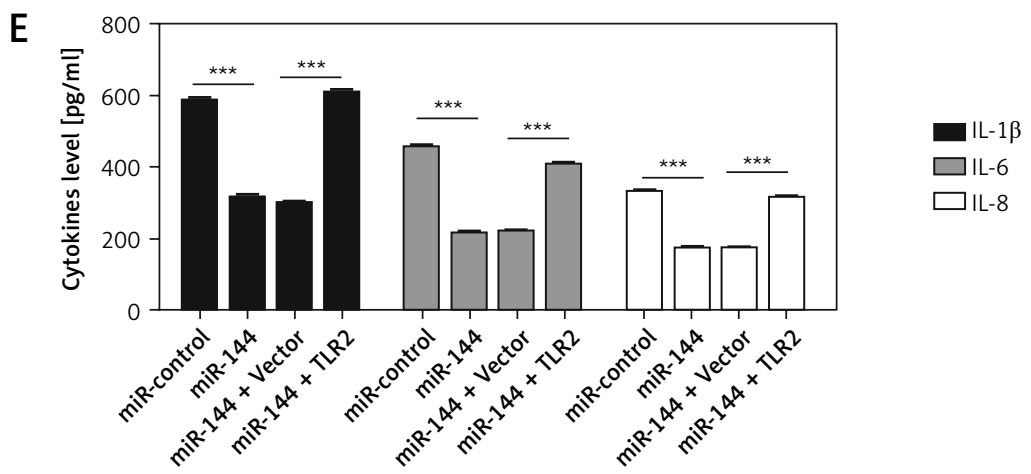

Figure 6. Inhibitory effects of miR-144 on proliferation, migration, invasion and inflammatory cytokine release of A549 cells were reversed by TLR2 overexpression. A549 cells were co-transfected with miR-144 mimic and TLR2 lacking 3'UTR or control vector. After transfection for 48 h. A - Expression of miR-144 was detected by qPCR. B - Expression of TLR2 was detected by western blot. C - Cell migration was detected by wound healing assay. D - Cell invasion was detected by transwell invasion assay. E - Inflammatory cytokine secretion was detected by ELISA. ${ }^{*} P<0.05,{ }^{* *} p<0.01,{ }^{* *} p<0.001$

been reported that miR-144 inhibits tumor cell migration, invasion and EMT-mediated metastasis by directly targeting proline-rich protein 11 in pancreatic cancer [24], c-Met in multiple myeloma [25], ZFX in hepatocellular carcinoma [26] and PBX3 in gastric cancer [27]. To elucidate the underlying mechanisms of miR-144 mediated inhibitory effects on NSCLC migration, invasion and inflammatory cytokine release, we used
TargetScan to predict the gene target of miR-144. TLR2 was identified as a key target gene of miR144. We further confirmed this prediction by luciferase reporter assay. In addition, upregulation of miR-144 significantly reduced protein levels of TLR2 in NSCLC cells.

Chronic inflammation is involved in many pathological processes including chronic venous leg ulcer (CVU), coronary artery disease (CAD) and 
acute myocardial infarction [28-31]. Moreover, chronic inflammation modulates almost all aspects of tumor behavior [7]. TLRs also play a key role in regulation of chronic inflammation and immune tolerance [32]. Emerging data have demonstrated that TLRs are involved in the progression and development of cancer [33]. Knockdown of TLR2 in H22 tumor cells abrogated the growth promoting effects of Listeria monocytogenes [34]. TLR2 can regulate the production of inflammatory cytokines such as IL-6, IL-1 $\beta$ and IL- 8 that can promote tumor cell migration and invasion [20, 35]. It was reported that TLR2 was overexpressed in NSCLC [36], which is in accordance with our observations. However, the role of TLR2 in NSCLC is not clear. Here, we found that down-regulation of TLR2 in A549 inhibits migration, invasion as well as inflammatory cytokine and chemokine release of NSCLC cells. Moreover, the inhibitory effects of miR-144 on NSCLC cell migration, invasion and inflammatory cytokine release were reversed by TLR2 overexpression. Thus, the mechanism of miR-144 mediated inhibitory effects on NSCLC migration and invasion may lie in its inhibitory functions on TRL2 expression and inflammatory cytokine release.

In conclusion, our results showed that miR-144 is significantly downregulated in NSCLC tissues and cell lines. Overexpression of miR-144 suppressed cell migration, invasion and inflammatory cytokine release of NSCLC through directly targeting TLR2. These data suggest that the frequently downregulated miR-144 leads to the upregulation of TLR2 and in turn contributes to the development of NSCLC.

\section{Conflict of interest}

The authors declare no conflict of interest.

\section{References}

1. Jemal A, Siegel R, Ward E, et al. Cancer statistics, 2008. CA Cancer I Clin 2008; 58: 71-96.

2. Mitsudomi T, Suda K, Yatabe Y. Surgery for NSCLC in the era of personalized medicine. Nat Rev Clin Oncol 2013; 10: 235-44.

3. Verdecchia A, Francisci S, Brenner $\mathrm{H}$, et al. Recent cancer survival in Europe: a 2000-02 period analysis of EUROCARE-4 data. Lancet Oncol 2007; 8: 784-96.

4. Wu F, Xu J, Huang $\mathrm{Q}$, et al. The role of interleukin-17 in lung cancer. Mediators Inflamm 2016; 2016: 8494079.

5. Hong JT, Son DJ, Lee CK, Yoon DY, Lee DH, Park MH. Interleukin 32, inflammation and cancer. Pharmacol Ther 2017; 174: 127-37.

6. Cho WC, Kwan CK, Yau S, So PP, Poon PC, Au JS. The role of inflammation in the pathogenesis of lung cancer. Expert Opin Ther Targets 2011; 15: 1127-37.

7. DeCotiis C, Hu Y, Greenberg AK, et al. Inflammatory cytokines and non-small cell lung cancer in a CT-scan screening cohort: background review of the literature. Cancer Biomark 2016; 16: 219-33.
8. Sabroe I, Read RC, Whyte MK, Dockrell DH, Vogel SN, Dower SK. Toll-like receptors in health and disease: complex questions remain. J Immunol 2003; 171: 1630-5.

9. O'Callaghan DS, O'Donnell D, O'Connell F, O'Byrne KJ. The role of inflammation in the pathogenesis of nonsmall cell lung cancer. J Thorac Oncol 2010; 5: 2024-36.

10. Li Q, Han Y, Fei G, Guo Z, Ren T, Liu Z. IL-17 promoted metastasis of non-small-cell lung cancer cells. Immunol Lett 2012; 148: 144-50.

11. Yao X, Huang J, Zhong $\mathrm{H}$, et al. Targeting interleukin- 6 in inflammatory autoimmune diseases and cancers. Pharmacol Ther 2014; 141: 125-39.

12. Guo H, Ingolia NT, Weissman JS, Bartel DP. Mammalian microRNAs predominantly act to decrease target mRNA levels. Nature 2010; 466: 835-40.

13. Filkova M, Jungel A, Gay RE, Gay S. MicroRNAs in rheumatoid arthritis: potential role in diagnosis and therapy. BioDrugs 2012; 26: 131-41.

14. Vasilatou D, Papageorgiou S, Pappa V, Papageorgiou E, Dervenoulas J. The role of microRNAs in normal and malignant hematopoiesis. Eur J Haematol 2010; 84: 1-16.

15. Akiyoshi S, Fukagawa T, Ueo H, et al. Clinical significance of miR-144-ZFX axis in disseminated tumour cells in bone marrow in gastric cancer cases. Br J Cancer 2012; 107: 1345-53.

16. Guo Y, Ying L, Tian Y, et al. miR-144 downregulation increases bladder cancer cell proliferation by targeting EZH2 and regulating Wnt signaling. FEBS J 2013; 280: 4531-8.

17. Iwaya T, Yokobori T, Nishida N, et al. Downregulation of miR-144 is associated with colorectal cancer progression via activation of mTOR signaling pathway. Carcinogenesis 2012; 33: 2391-7.

18. Gao F, Wang T, Zhang Z, Wang R, Guo Y, Liu J. Regulation of activating protein-4-associated metastases of nonsmall cell lung cancer cells by miR-144. Tumour Biol 2015. doi: 10.1007/s13277-015-3866-4.

19. Yang CW, Hung CC, Wu MS, et al. Toll-like receptor 2 mediates early inflammation by leptospiral outer membrane proteins in proximal tubule cells. Kidney Int 2006; 69: 815-22.

20. Sugitharini V, Shahana P, Prema A, Berla Thangam E. TLR2 and TLR4 co-activation utilizes distinct signaling pathways for the production of Th1/Th2/Th17 cytokines in neonatal immune cells. Cytokine 2016; 85: 191-200.

21. Peng JS, Chen SY, Wu CL, et al. Amelioration of experimental autoimmune arthritis through targeting of synovial fibroblasts by intraarticular delivery of microRNAs 140-3p and 140-5p. Arthritis Rheumatol 2016; 68: 370-81.

22. Yanaihara N, Caplen N, Bowman E, et al. Unique microRNA molecular profiles in lung cancer diagnosis and prognosis. Cancer Cell 2006; 9: 189-98.

23. Ritchie W, Rasko JE, Flamant S. MicroRNA target prediction and validation. Adv Exp Med Biol 2013; 774: 39-53.

24. Li J, Sun P, Yue Z, Zhang D, You K, Wang J. miR-144-3p induces cell cycle arrest and apoptosis in pancreatic cancer cells by targeting proline-rich protein 11 expression via the mitogen-activated protein kinase signaling pathway. DNA Cell Biol 2017; 36: 619-26.

25. Zhao Y, Xie Z, Lin J, Liu P. MiR-144-3p inhibits cell proliferation and induces apoptosis in multiple myeloma by targeting c-Met. Am J Transl Res 2017; 9: 2437-46.

26. Bao H, Li X, Li H, et al. MicroRNA-144 inhibits hepatocellular carcinoma cell proliferation, invasion and migration by targeting ZFX. J Biosci 2017; 42: 103-11.

27. Li B, Zhang S, Shen H, Li C. MicroRNA-144-3p suppresses gastric cancer progression by inhibiting epitheli- 
al-to-mesenchymal transition through targeting PBX3. Biochem Biophys Res Commun 2017; 484: 241-7.

28. Moreno-Eutimio MA, Espinosa-Monroy L, Orozco-Amaro $T$, et al. Enhanced healing and anti-inflammatory effects of a carbohydrate polymer with zinc oxide in patients with chronic venous leg ulcers: preliminary results. Arch Med Sci 2018; 14: 336-44.

29. Stumpf C, Sheriff A, Zimmermann S, et al. C-reactive protein levels predict systolic heart failure and outcome in patients with first ST-elevation myocardial infarction treated with coronary angioplasty. Arch Med Sci 2017; 13: 1086-93.

30. Caglar FNT, Isiksacan N, Biyik I, Opan S, Cebe H, Akturk IF. Presepsin (sCD14-ST): could it be a novel marker for the diagnosis of ST elevation myocardial infarction? Arch Med Sci Atheroscler Dis 2017; 2: e3-e8.

31. Trocha M, Merwid-Lad A, Piesniewska M, et al. Agerelated differences in function and structure of rat livers subjected to ischemia/reperfusion. Arch Med Sci 2018; 14: 388-95.

32. de Visser KE, Eichten A, Coussens LM. Paradoxical roles of the immune system during cancer development. Nat Rev Cancer 2006; 6: 24-37.

33. Rakoff-Nahoum S, Medzhitov R. Toll-like receptors and cancer. Nat Rev Cancer 2009; 9: 57-63.

34. Kluwe J, Mencin A, Schwabe RF. Toll-like receptors, wound healing, and carcinogenesis. J Mol Med (Berl) 2009; 87: 125-38.

35. Storr SJ, Safuan S, Ahmad N, El-Refaee M, Jackson AM, Martin SG. Macrophage-derived interleukin-1beta promotes human breast cancer cell migration and lymphatic adhesion in vitro. Cancer Immunol Immunother 2017; 66: 1287-94.

36. Yu L, Chen S. Toll-like receptors expressed in tumor cells: targets for therapy. Cancer Immunol Immunother 2008; 57: 1271-8. 